\title{
Automated system for synthesis of sensors for smart cities
}

\author{
Irina Petrova ${ }^{1,}$, Viktoriya Zaripova ${ }^{1}$, Yuliya Lezhnina ${ }^{1, *}$ and Ilkhomzhon Siddikov ${ }^{1,2}$ \\ ${ }^{1}$ Astrakhan state university of architecture and civil engineering, Department of CAD and modelling \\ systems, 414056 Taticheva str., 18, Russia \\ ${ }^{2}$ Tashkent University of Information Technologies, Production Department, 100200, Amir Temur str., \\ 108, Uzbekistan
}

\begin{abstract}
The concept of a smart city is aimed at developing technologies and infrastructure based on the use of new technologies, large data centers, smart sensors and automated power grids. As technological advances in control systems increase the efficiency of sensors, users are calling for advances in sensor accuracy, reliability, dynamic characteristics (response time, dynamic linearity etc.), robustness, miniaturization and communication capability. The sensor industry is expected to experience sound growth during the five-year forecast period. It is possible satisfy the growing demand, in particular, by using automated systems of conceptual design of sensors, which can reduce the design time several times. Physical principles of operation of the sensors involved in information measuring and control systems of buildings are quite diverse. In this article, it is proposed to use a modified energy-information method for the conceptual design of sensors, invariant to the physical principle of operation. The possibility of using the operator method for the calculation and study of the dynamic characteristics of the designed elements of control systems of different physical nature is shown. The architecture of the expert system of evaluation of dynamic characteristics of sensitive elements of informationmeasuring devices of smart home is developed.
\end{abstract}

\section{Introduction}

The emergence of a "smart city" is associated with the development of public services [1] by optimal management of the urban transport network [2], rational use of energy [3, 4], improving the quality of health care [5,6] rational use of water [7] and other resources, and an innovative approach to agriculture and waste management. The "smart city" is a combination of modern technologies and solutions, which allows improving the quality of life of citizens and, at the same time, help the city economy more efficiently use available resources [8]. The progress of the Smart Cities is based on the introduction of new ways, when, thanks to sensors and algorithms, the control and management capabilities are enhanced $[9,10]$. Sensors have become indispensable in many industries because they

\footnotetext{
* Corresponding author: lejninau@gmail.com
} 
provide vital information about parameters that include temperature, position, chemistry, pressure, force and load, flow and level, and thus affect products, processes and systems.

As technological advances in control systems increase the efficiency of sensors, users are calling for advances in sensor accuracy, reliability, dynamic characteristics (response time, dynamic linearity etc.), robustness, miniaturization and communication capability. The sensor industry is expected to experience sound growth during the five-year forecast period. Technavio has published a new market research report on the global industrial sensors market for the period 2018-2022. The industrial sensors market size will grow by over USD 17 billion during 2018-2022 [11].

Growing market of smart cities, deployment of smart sensors in healthcare sector and increasing environment safety concern are expected to drive the market during the forecast period 2017-2023. However, high development cost of smart sensors is the major factor hindering the market growth. To meet growing demand, in particular, using automated systems for conceptual design of sensors $[12,13]$. Such systems, as is known, can reduce the design time by several times.

\section{Statement of the problem}

The control system of a smart city is an infrastructure with a multitude of sensors and video cameras integrated through wireless broadband networks. Through the Internet of Things (IoT), such different systems as fire protection, climate control, ventilation and video surveillance can be combined into a single network. Creating new miniature sensors and actuators will support the concept of a smart city - equipped with millions of sensors that collect information about everything that happens on the streets and in homes. The dynamic characteristics of the sensor are critical for smart cities control systems. These characteristics cannot be changed after manufacture of the sensor. Therefore, it is important at the initial stage of the conceptual design of the sensor to evaluate its dynamic characteristics.

There is a fairly large number of conceptual design methods for new technical solutions based on knowledge bases of physical effects and phenomena. [14, 15, 16].

All these methods are based on classified knowledge of physical effects. But all of them are aimed at support of conceptual design of any technical devices regardless of the sphere of its application.

Conceptual design of sensing elements (sensors) for data measurement and control systems has some peculiar features. On the one hand it is possible to design fairly simple structural diagrams reflecting the principle of the sensor operation; on the other hand, input of at least one new phenomenon to the knowledge base generates many new principles of sensor operation.

Energy-information model of circuits (EIMC) enables switching to structural and formalized description of processes of sensor elements by using parametric structural schemes $[12,13]$. The operation principle of any element of data measurement and control systems is based on interaction of circuits of different physical nature, which is simulated in EIMC by the sequence of physical and technical effects.

The development of conceptual model of knowledge systematization of physical phenomena and effects for new sensitive elements synthesis is based on principles of nonbalanced thermodynamics $[17,18]$ because they enable to get the full system of transfer equations and other regularities, not revealing their molecular mechanism. The authors suggested energy-information models of processes of various physical nature (EIMC) on the base of phenomenological equations of non-balanced thermodynamics, gave the main definitions of EIMC. 
The main definitions of this model (EIMC) as well as quantities and parameters were given in $[12,13]$. These quantities and parameters are related with equations named "EIMC criteria" by the authors.

They are given in $[12,13]$ for a simple case of constant parameters systems. Principles of action of many modern sensors are based on the processes of self-oscillation, parametrical amplification, and others with different physical nature parameters changeable in time. Therefor the authors introduced additional quantities characterizing speed of main EIMC quantities change, and enlarged a set of main criteria for the systems with changeable in time parameters (tabl.1). Using the basics of the Laplase transform to calculate the transfer function of a complex parametric structural schemes allows you to explore the dynamic characteristics of the designed elements of control systems of various physical nature. The knowledge base of the conceptual design system stores all possible combinations of transfer functions for two and three elementary units.

Table 1. Set of main criteria for the systems with dynamic parameters (the Laplace form: p-domain).

\begin{tabular}{|c|c|c|c|c|c|}
\hline EIMC & \multicolumn{5}{|c|}{ Parameters in the Laplace form } \\
\hline & criterion & parameter & Input value & Scheme & \\
\hline Static & $U(p)=R(p) \cdot I(p)$ & $R(p)$ & $I(p)$ & $\stackrel{I(p)}{\longrightarrow} R(p$ & $\underset{\longrightarrow}{U(p)}$ \\
\hline Static & $I(p)=G(p) \cdot U(p)$ & $G(p)$ & $U(p)$ & $\stackrel{U(p)}{\longrightarrow} G(p$ & $\stackrel{I(p)}{\longrightarrow}$ \\
\hline Dinamic I & $U(p)=(W / p) \cdot I(p)$ & $W(p)=W / p$ & $I(p)$ & $\stackrel{I(p)}{\rightarrow} \quad W$ & $\underset{\longrightarrow}{\longrightarrow}(p)$ \\
\hline Dinamic I & $I(p)=p C \cdot U(p)$ & $C(p)=p C$ & $U(p)$ & $\stackrel{U(p)}{\longrightarrow} C(p$ & $\stackrel{I(p)}{\longrightarrow}$ \\
\hline Dinamic II & $U(p)=p L \cdot I(p)$ & $L(p)=p L$ & $I(p)$ & $\stackrel{I(p)}{\longrightarrow} L(p$ & $U(p)$ \\
\hline Dinamic II & $I(p)=(D / p) \cdot U(p)$ & $D(p)=D / p$ & $U(p)$ & $\stackrel{U(p)}{\longrightarrow} D(p$ & $\stackrel{I(p)}{\longrightarrow}$ \\
\hline
\end{tabular}

Using energy-information models for describing chains of different physical nature and the apparatus of parametric structural schemes $[12,13]$, one can express the whole diversity of interrelations between quantities and parameters of different physical nature $(i$ or $j)$ as a complex graph (Fig. 1). This article proposed to introduce elements in this graph reflecting dynamic processes in the form of Laplace transforms. (the Laplace transform) [17]:

- parameters $R_{i}, G_{i}, p C_{i}, W_{i} / p, p L_{i}, D_{i} / p$,

- values $Q_{i}(p), P_{i}(p), U_{i}(p), I_{i}(p)$,

- differentiation elements - $p$ and integration $-1 / p$.

Here $p$ - the Laplace operator.

The vertices of the graph - the magnitude of various physical nature, and the edges circuit parameters of the physical nature (electrical, magnetic, thermal, mechanical, etc.). In the general case, we can assume that all of them are represented by their images in the operator form. The connection between the chains of different physical nature is carried out 
with the help of physico-technical effects, the coefficients of which $\left(K_{i j}\right.$ or $\left.K_{j i}\right)$ in a simplified form can be considered quasi-permanent.

Using the graph, it is possible to determine from the given input and output values of the projected sensor possible variants of the physical principle of operation (i.e., the path along the graph). For each physical effect or parameter, the knowledge base stores sets of averaged values of operational characteristics. Therefore, using the obtained parametric structural schemes, it is possible to calculate the static and dynamic performance characteristics of the entire circuit as a whole (sensitivity, reliability, speed, etc.).

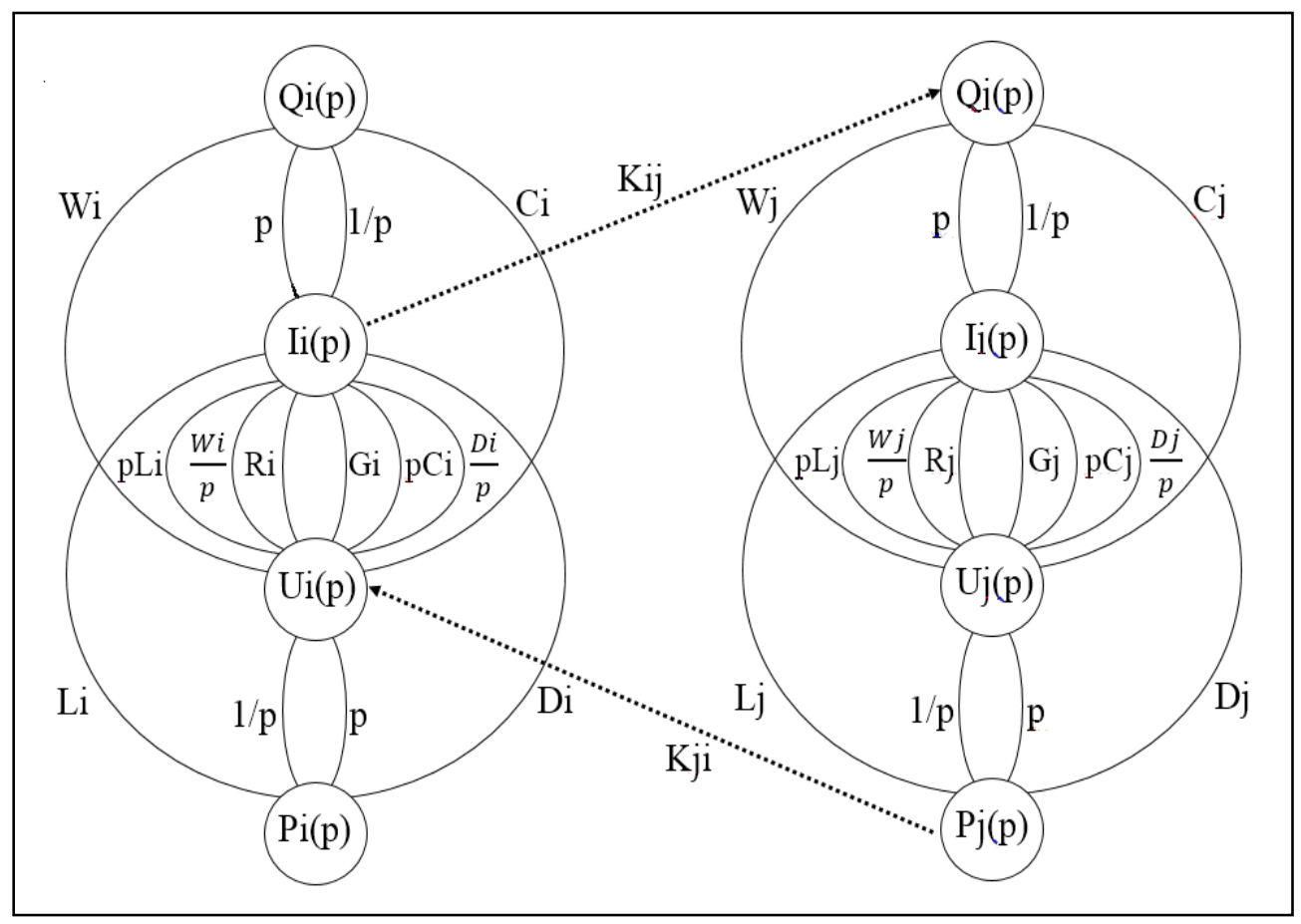

Fig. 1. The graph of the relationship between quantities and parameters in the operator form for chains of different physical nature (i-th and $j$-th nature)

General properties of the task are as follows:

- there is a finite set of options for the synthesis of parametric structural schemes, reflecting the various physical principles of the sensor with given input and output;

- each variant of the parametric structural scheme is associated with a set of calculated values of operational characteristics (sensitivity, reliability, accuracy, range of input and output values, degree of nonlinearity of the output characteristic, speed, etc.). They are calculated by the values of the characteristics of individual units (physical and technical effects or parameters);

- it is required to choose a variant of the physical principle of the sensor operation on the basis of a set of operational characteristics that meets certain pre-formulated requirements.

Thus, the task is reduced to the task of complete enumeration using the strategy with the return. As a result, we obtain chains of successive transformations, reflecting the dynamics of the processes taking place. 


\section{Solution method}

To evaluate the dynamic characteristics, it is necessary to first find the analytical form of the transfer function using a parametric block diagram and Laplace transform method, and then move from the image to the original function and get the time dependence (Inverse Laplace Transform).

To obtain the expression of the transfer function via Laplace transform for the synthesized parametric block diagram, a universal algorithm based on the Mason's formula $[20,21]$ was used. Mason's formula allows a graphical image of a parametric structural diagram to convert, to the oriented graph of this scheme and determine the general formula transfer function in operator form (p-domain). To calculate the transfer function of the parametric block diagram using the Mason formula, the following set of algorithms was elaborated:

1) Algorithm of search all single ways in graph,

2) Algorithm of search all single cycles,

3) Algorithm of search all sets of "untouchment" cycles,

4) Algorithm of search all single cycles in subgraph.

To move transfer function of the parametric block diagram from the $\mathrm{p}$-domain to the $\mathrm{t}$ domain (inverse Laplace transform), need to perform the following steps and algorithms:

1. Algorithm Find_Roots - it find the roots of the denominator of the output transfer function using the Fletcher-Reeves algorithm [20-23].

2. The Solve_Matrix algorithm implements the decomposition of the transfer function into elementary addend: $A /(p-a)$ and $(d+e \cdot d) /(p-c \cdot j)$. Moreover, the terms of the corresponding complex-conjugate roots are combine and merge:

$$
\frac{d+e \cdot j}{p-(b+c \cdot j)}+\frac{d-e \cdot j}{p-(b-c \cdot j)}=\frac{2 \cdot d \cdot p+(-2 \cdot b \cdot d-2 \cdot e \cdot c)}{p^{2}-2 \cdot b \cdot p+\left(b^{2}+c^{2}\right)}=\frac{A \cdot p+B}{p^{2}+a_{1} \cdot p+a_{2}}
$$

3. Transfer from p-domain to t-domain (inverse Laplace transform) is as follows:

$$
\frac{A \cdot p+B}{p^{2}+a_{1} \cdot p+a_{2}} \rightarrow e^{-\frac{a_{1}}{2} \cdot t} \cdot\left[A \cdot \cos \left(\sqrt{a_{2}-\frac{a_{1}^{2}}{4}} \cdot t\right)+\frac{B-\frac{A \cdot a_{1}}{2}}{\sqrt{a_{2}-\frac{a_{1}^{2}}{4}}} \cdot \sin \left(\sqrt{a_{2}-\frac{a_{1}^{2}}{4}} \cdot t\right)\right]
$$

\section{Architecture of the automated system for synthesis of the operating prin- ciple of sensors with the assessment of dynamic characteristics}

The article considers conceptual model of a system of new technical solutions searching for assessing the dynamic characteristics of sensitive elements of information-measuring devices. Automated system allows users to explore the scheme, built on the basis of elementary blocks and blocks of the type "Variable parameter" of energy-information model.

The system provides for the interaction of three actors (administrator, expert and engineer-end-user of the system) (Fig.2). The administrator differentiates the rights to use 
the system, allowing the expert to modify the physical effects passports and parameters



available in the knowledge base, as well as create new passports.

Fig. 2. Diagram of use cases of the automated system of support of conceptual design of IMCS elements

Expert and engineer after registration on the website have the opportunity to work with the system. The expert maintains the knowledge base in the current state: replenishes the database of physical effects passports and parameters, updates the ball scores of performance in the passports, etc. The Engineer - the end user of the system, after registration, has the opportunity to synthesize new technical solutions in the form of parametric structural schemes, to calculate the performance characteristics of the synthesized parametric block diagrams and rank them according to the totality of these characteristics, to save the obtained solutions. In addition, according to the synthesized block diagram, the engineer can study the dynamic characteristics using the developed subsystem of automated calculation of dynamic characteristics. 
The subsystem for calculating dynamic characteristics (Fig. 3) allows calculating the dynamic characteristics of a parametric block diagram (output temporal characteristic, amplitude-frequency, phase-frequency and complex-frequency characteristic) and organizes the interface to the synthesis program.

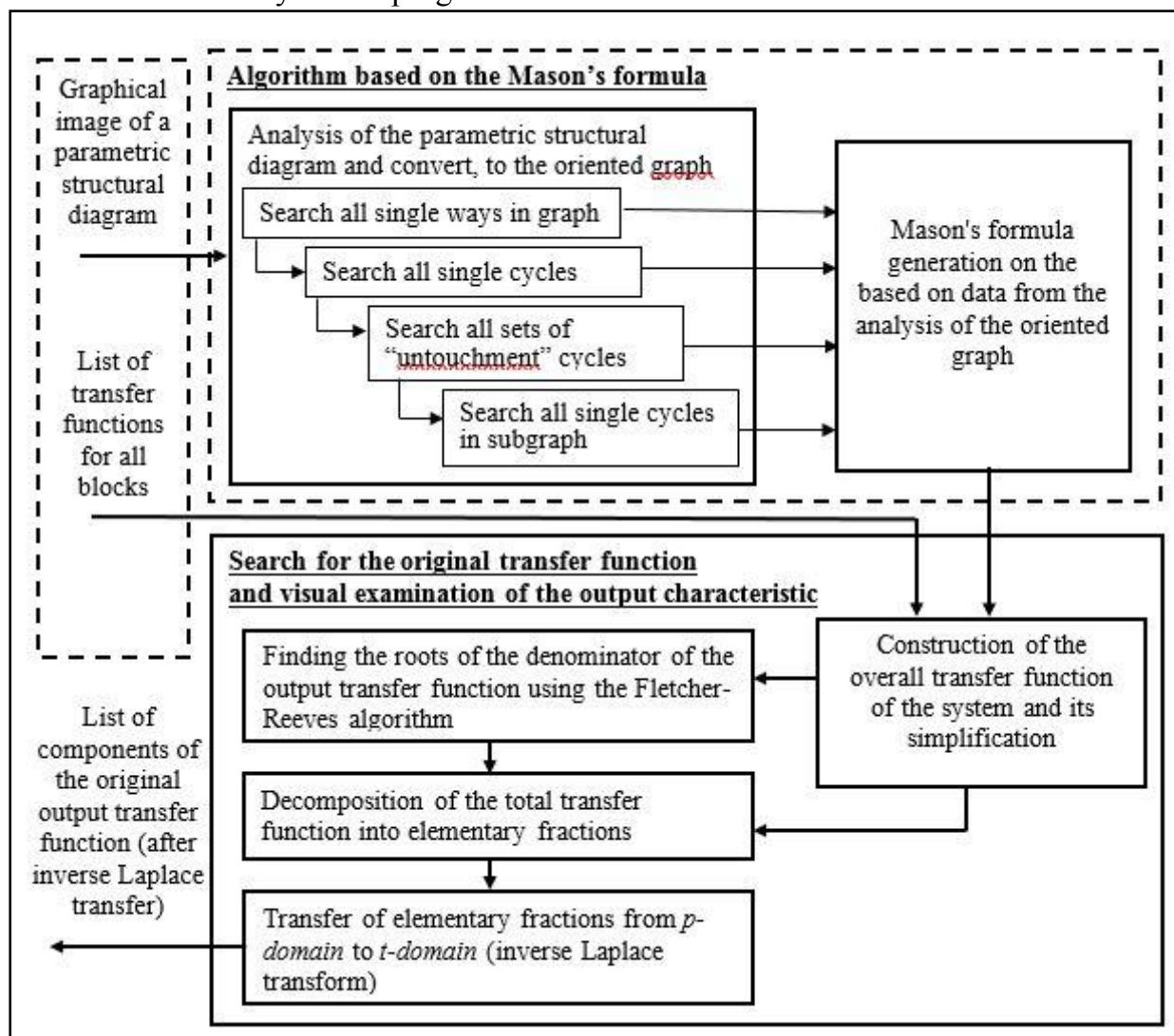

Fig. 3. Diagram of information flows in the subsystem for calculating dynamic characteristics.

In fig. 4. shows a test example of a parametric block diagram. As a result of the theoretical calculation of this scheme, the following output function was obtained manually $f(t)=-1,32 \cdot e^{-2,64 t}+1,32 \cdot e^{-1,14 t}$. This completely coincides with the results obtained after the calculation using an automated system (Fig. 4.)

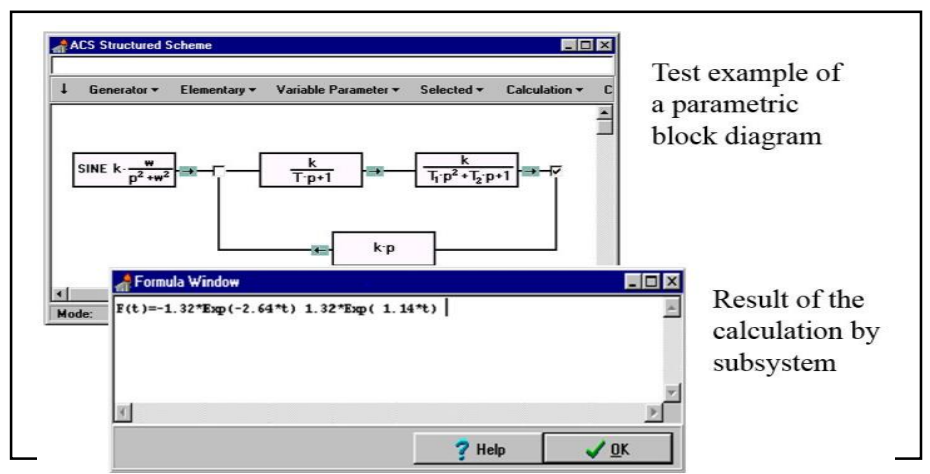

Fig. 4. An example of the calculation of the dynamic characteristics of a parametric block diagram. 
The complex-frequency characteristic calculated manually can be represented by the formula: $W(j \omega)=10 \omega x+30(2 \omega x+6)+(-3 \omega)$ j $15 \omega(2 \omega$

$$
W(j \varpi)=\frac{10 \cdot \varpi x^{4}+30}{\left(2 \cdot \varpi^{2}+6\right)^{2}+(-3 \cdot \varpi)^{2}}+j \cdot \frac{15 \cdot \varpi}{\left.2 \cdot \varpi^{2}+6\right)^{2}+(-3 \cdot \varpi)^{2}}
$$

This formula coincides with the formula obtained using the subsystem (Fig. 5.)

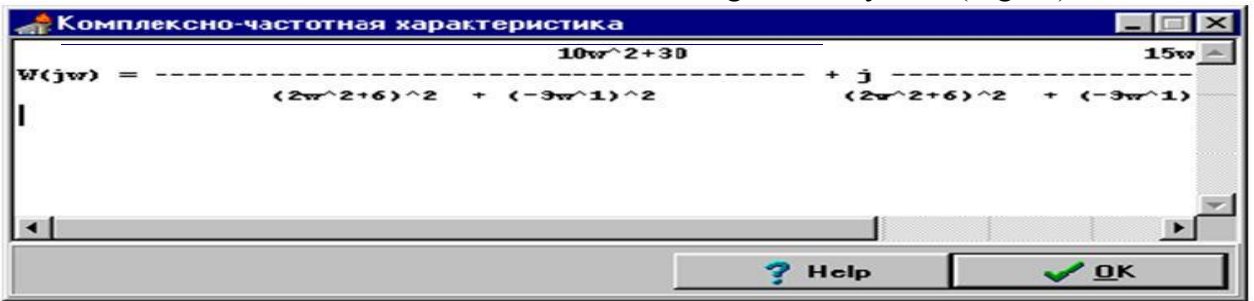

Fig. 5. The result of the calculation of the complex-frequency characteristics using an subsystem for calculating dynamic characteristics

\section{Conclusion}

Analysis of the well-known systems of conceptual design of elements of Information Measuring and Control Systems showed that most of them are distinguished by narrow specialization and high requirements for the user's initial knowledge.

Energy-informational models for the description of chains of various physical nature and the apparatus of parametric structural schemes make it possible to represent the whole variety of interrelations between quantities and parameters in the form of a complex graph. For the first time, elements reflecting dynamic processes in operator form were introduced into this graph.

To find the transfer function of a complex structural scheme in operator form, a universal algorithm has been developed using the Mason formula, which allows a graphical image of a parametric structural scheme to go to the oriented graph of this scheme and determine the general formula of the image using the Laplace output characteristic.

To solve the problem of transition from the image of the transfer function of a complex structural scheme to the original, to further study the dynamic characteristics, it was proposed to use the Fletcher-Reeves algorithm, which allows finding the roots of the denominator of the output transfer function and decompose it into elementary terms.

The developed models, methods and algorithms are implemented in the form of a complex of programs for the synthesis and analysis of the dynamic characteristics of the elements of information-measuring and control systems. The use of the developed complex makes it possible to reduce the time for conceptual design by $1.5-2$ times.

A design engineer with the support of a conceptual design system can satisfy the requirements for minimizing sensors and actuators, expand their functionality, and ensure cheaper production. Thus, the technical component of the concept of a smart city will be effectively developed.

\section{References}

1. F. De Filippi et al., IEEE Access, 4, 3824-3843 (2016)

2. B. Lenz and D. Heinrichs, IEEE Pervasive Computing, 16(2), 84-86 (2017) 
3. L. Liu, Y. Liu, L. Wang, A. Zomaya and S. Hu, IEEE Transactions on Emerging Topics in Computing, 3(4), 556-570 (2015)

4. R. Carli, M. Dotoli and R. Pellegrino, IEEE Transactions on Automation Science and Engineering, 14(2), 505-523 (2017)

5. D. J. Cook, G. Duncan, G. Sprint and R. L. Fritz, Proceedings of the IEEE, 106(4), 708-722, (2018)

6. J. Maria de Fuentes, L. Gonzalez-Manzano, A. Solanas and F. Veseli, Computer, 51(7), 44-53 (2018)

7. P. Kulkarni and T. Farnham, IEEE Access, 4, 660-672 (2016)

8. A. Volkov, O. Kuzina, Procedia Engineering, 153, 838-843 (2016)

9. Vytovtov, K.A., Barabanova, E.A., Barabanov, I.O. International Conference on Actual Problems of Electron Devices Engineering, 1, 306-310 (2018)

10. I. Jabłoński, IEEE Sensors Journal, 17(23), 7659-7666, (2017)

11. Global Industrial Sensors Market 2018-2022 // URL: https://www.technavio.com/report/global-industrial-sensors-market-analysis-share2018 (14.11.2018)

12. V. Zaripova, I. Petrova, Series: Advances in Intelligent Systems and Computing, 1089, 365-373 (2015)

13. V. M. Zaripova and I. Yu. Petrova, JoP: Conference Series 588, 012031, (2015)

14. R. Koller, Konstruktionslehre für den Maschinenbau. Grundlagen des methodischen Konstruierens (Berlin, Springer Vlg., Heidelberg: NY, 1985)

15. G. Altshuller, And Suddenly the Inventor Appeared - TRIZ (Technical Innovation Center, Worcester MA, 2008)

16. K. Rantanen, E. Domb, Simplified TRIZ: New Problem Solving Applications for Engineers and Manufacturing Professionals (Taylor \& Francis Ltd, 2007)

17. S. R. De Groot and P. Mazur, Non-Equilibrium Thermodynamics (North-Holland Publishing Company, Amsterdam, 1962).

18. I. Prigogine, Introduction to thermodynamics of irreversible processes (Interscience Publishers, New York, 1968).

19. G. A. Korn, and T.M. Korn, Mathematical Handbook for Scientists and Engineers. Definitions, Theorems, and Formulas for Reference and Review (Dover Publications, Inc., New York, 2000)

20. S. N. Verma, Automatic Control system (Khanna Publishers, New Delhi, 2006)

21. V. C. Prasad, International Journal of Electrical Engineering Education, 47(4), 393-403 (2010)

22. R. Fletcher, C. M. Reeves, Computer Journal, 7, 149-54 (1964)

23. Michiel Hazewinkel, Conjugate gradients, method of", Encyclopedia of Mathematics (Springer Science+Business Media B.V., Kluwer Academic Publishers, 1994) 